\title{
Fluorescence depletion mechanisms in super-resolving STED microscopy
}

\author{
E. Rittweger, B.R. Rankin, V. Westphal *, S.W. Hell \\ Max Planck Institute for Biophysical Chemistry, Department of NanoBiophotonics, Göttingen, Germany
}

Received 22 February 2007; in final form 29 May 2007

Available online 8 June 2007

\begin{abstract}
We prove that stimulated emission is by far the dominant quenching mechanism for providing super-resolution in fluorescence microscopy with a red-shifted depletion beam. Our evidences are based on simultaneously measuring fluorescence quenching and photon gain in the quenching beam. Measurements were performed for several fluorescent dyes including fluorescent proteins over a wide spectral range of their emission spectra. We found that, for each fluorophore, the wavelength dependence of both signals closely follows that of the stimulated emission cross-section.
\end{abstract}

(C) 2007 Published by Elsevier B.V.

\section{Introduction}

Stimulated emission depletion (STED) microscopy has been developed over the last decade into a powerful tool for biological imaging, providing spatial resolution well beyond the diffraction limit, so far up to ten fold $[1,2]$. This is achieved by employing a diffracted, focused laser pulse that excites the fluorescent molecules in the sample. Excitation is followed by a second pulse with a doughnut-shaped intensity profile that de-excites the molecules in the periphery of the excitation focal spot, allowing only the fluorescence from the molecules in the very center to contribute to the image. Many spectroscopic studies of laser dyes and biological fluorescent markers support that this deexcitation process is realized via stimulated emission [37]. However, the photophysics of such dyes, as applied to fluorescence quenching in microscopy, is still controversial.

Several recent papers [8-11] have proposed an alternative process, termed up-conversion, by which excited molecules are quenched by the doughnut-shaped beam: the molecules are further excited from $S_{1}$ to higher excited singlet states $S_{n}$, from which they relax back to $S_{1}$ via vibra-

\footnotetext{
* Corresponding author. Fax: +49 5512012505.

E-mail address: vwestph@gwdg.de (V. Westphal).
}

tional relaxation or internal conversion. If several photons are absorbed by the same molecule during the second pulse, the molecule can be excited from $S_{1}$ several times, each time relaxing back to $S_{1}$ vibrationally and dissipating heat into its local environment [12]. This heating could lower the quantum efficiency of the dye (e.g. by $10 \%$ for Rhodamine $6 \mathrm{G}$ for $\Delta T=40{ }^{\circ} \mathrm{C}$ [13]), causing it to relax non-radiatively to $\mathrm{S}_{0}$ via internal conversion. It is proposed that in this manner the fluorescence is quenched $[14,15]$.

The aim of this work is to clarify the question of which process, stimulated emission or up-conversion, is dominant in the quenching of fluorescence for a number of representative dyes emitting from the green to the near infrared end of the spectrum. We use a pump-probe technique to simultaneously measure both the fluorescence quenching and the change in number of photons in the probe beam. This allows us to distinguish between the possible dominant transitions which may be driven by the probe pulse after the molecules are excited to the $\mathrm{S}_{1}$ level. Fig. 1 shows the possible processes and how they would affect the two signals. Process (I), stimulated emission, would increase the number of photons in the probe beam and reduce the fluorescence signal. Process (II), excitation to higher levels and internal conversion to $S_{1}$, would remove a photon in the 


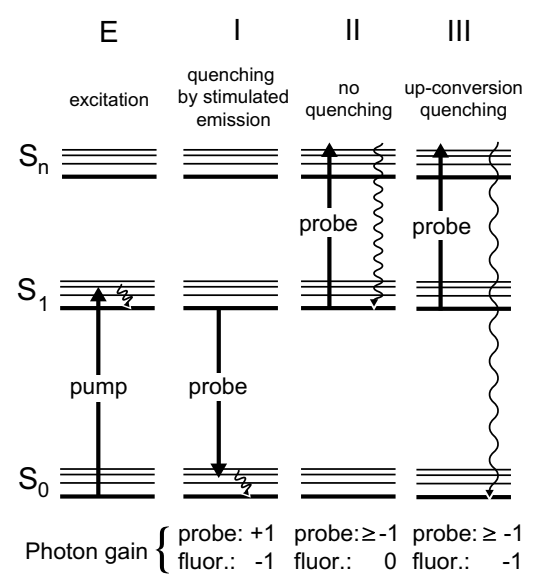

Fig. 1. Jablonski diagram showing three different possible transitions caused by interaction of the probe pulse after excitation (E): (I) Quenching by stimulated emission; (II) up-conversion without fluorescence quenching; (III) up-conversion with fluorescence quenching. Bottom: gain and loss of photons in probe beam and fluorescence light.

probe beam, but the fluorescence signal would not be affected since the molecule quickly $(<3 \mathrm{ps})$ returns to the $\mathrm{S}_{1}$ state. The last process (III), excitation to higher levels and internal conversion to $\mathrm{S}_{0}$, would reduce both signals.

Processes (I) and (III) would lead to fluorescence quenching. In order to break the diffraction barrier a strong, saturated depletion mechanism is required. Process (II) opens a possible channel for bleaching which is disastrous for resolution enhancement and image quality. As the up-conversion process would be a combination of multiple excited state absorption (II) and process (III), it is possible to discriminate the two possible depletion mechanisms clearly from one another depending on whether photons are gained or lost in the probe beam.

\section{Setup}

In Fig. 2 a schematic diagram of the experimental setup is depicted. The white-light spectrum of a supercontinuum fiber laser (Fianium, Southampton, UK) was divided by a dichroic mirror into the spectral parts A and B (See Fig. 2), from which the wavelengths for the pump and probe beams were selected using interchangeable bandpass filters (bandwidth $10 \mathrm{~nm}$; AHF Analysentechnik, Tübingen, Germany). The pulse lengths were around $100 \mathrm{ps}$. The repetition rate was $40 \mathrm{MHz}$. This design allows flexible measurements of different dyes over the whole visible range. Recombined by a second, identical dichroic mirror, the two collinear beams were focused into the cuvette with dye solution where the two foci superimpose. The intensity of the probe beam in the focus of $10^{4} \mathrm{~W} / \mathrm{cm}^{2}$ was some magnitudes smaller than the intensities used in STED microscopy $\left(\mathrm{GW} / \mathrm{cm}^{2}\right)$. However, this is not a problem, because in this study we are focusing on the cross-sections which are intrinsic properties of the dye molecules. The timing between the two pulses was controlled via a linear translation stage (Owis, Staufen, Germany). For the experiments

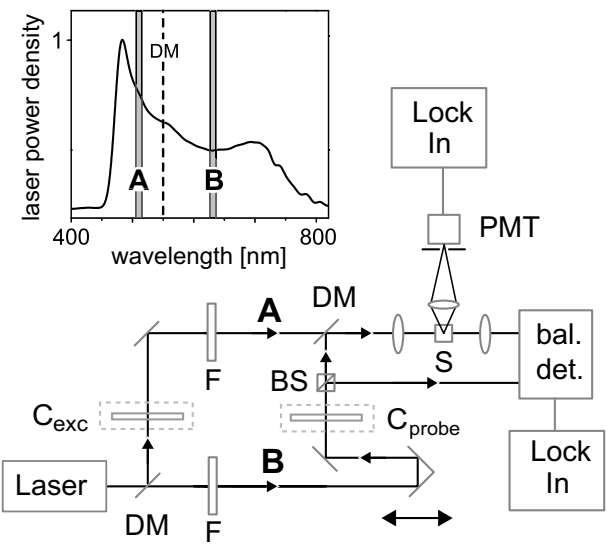

Fig. 2. Laser spectrum and setup; A: pump beam, B: probe beam, DM: dichroic mirror, $\mathrm{F}$ : bandpass filter, $\mathrm{C}_{\mathrm{exc}}$ : chopper position for measuring the change of the number of photons in the probe beam, $\mathrm{C}_{\text {probe }}$ : chopper position for fluorescence quenching measurement, $S$ : cuvette with dye solution, bal. det.: balanced detector, BS: beam splitter.

shown in Fig. 3 the delay between the pulses was chosen to yield the maximal depletion, roughly one pulse duration.

Two measurements were performed: the reduction of fluorescence and the change in the number of photons in the probe beam. In each case a small change in signal had to be discriminated from the baseline signal. Therefore a lock-in amplifier was used for noise reduction in both cases. For measuring the change in the number of photons in the probe and fluorescence quenching a chopper was placed in the excitation beam and the probe beam, respectively. To eliminate noise due to laser power fluctuations the probe beam was detected with an auto-balanced detector (Nirvana detector, New Focus, San Jose, USA) in comparison to a reference beam from the laser.

For both measurements one has to consider a background signal. In the gain measurements some fluorescence is inevitably detected which cannot be filtered out since the wavelength of the probe pulse lies within the emission band of the dye. Secondly, for the fluorescence quenching, an additional background can arise due to re-excitation induced by the probe beam. To be able to separate the signal of interest from the background it was measured while the probe pulse came ahead of the pump pulse. In this case no excited molecules were present when the probe pulse arrived. In this manner the pure background signal was measured. In order to assure a defined detection volume the fluorescence signal was imaged onto a pinhole in front of the detector. Thus the measurement was restricted to the region with the highest concentration of quenched molecules, which is the focus, the area of high light intensity and high density of excited molecules. A magnetic stirrer was used to increase the rate of dye solution exchange in the focal volume. This assures that the concentration of potentially bleached molecules or molecules in the triplet state can be neglected.

The two signals and their wavelength dependence were measured for eight fluorescent dyes covering different dye 


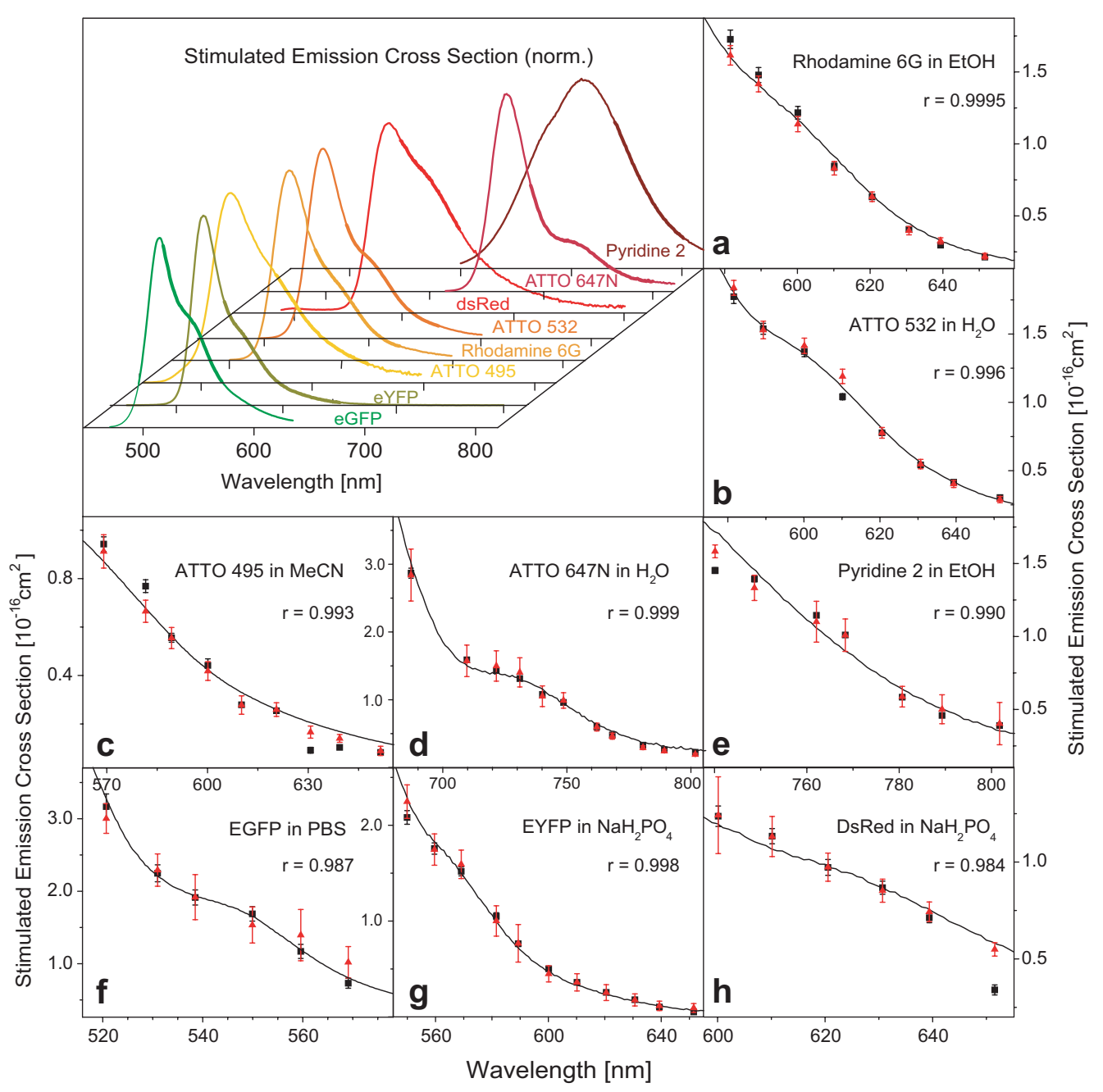

Fig. 3. Above left: Normalized stimulated emission cross-section for seven different dyes; the bold parts mark the areas which are shown in detail a-h: stimulated emission cross-section (thin line), relative measurement of STED gain ( $\boldsymbol{\square}$, black) and fluorescence quenching $(\boldsymbol{\Delta}$, red) and the values of their correlation. The results agree very well with each other which proofs that stimulated emission is the dominant process for fluorescence quenching.

families used in STED microscopy: the well-studied Rhodamine $6 \mathrm{G}$, in addition to ATTO 532, ATTO 495, ATTO 647, Pyridine 2 and three fluorescent proteins which are used for many biological applications: EGFP, EYFP and DsRed. ATTO 532 was used for many STED applications in the visible range $[2,16]$. EGFP and EYFP were recently used for the first time in a STED microscope [17]. The concentration of the dye solutions was about $200 \mu \mathrm{M}$. The solvents used are given in Fig. 3. The fluorescent proteins were expressed in Escherichia coli strain HMS 174 (DE3) using the expression plasmids pRSETa-EGFP, pRSETa-EYFP and pRSETa-DsRed. Cells expressing the protein were disrupted by Lysozyme treatment and sonication. For the presented measurements the whole protein extract was used.

\section{Results}

The fluorescence was quenched after switching on the probe pulse, which is consistent with observations applying the STED technique. Furthermore, photons were always gained in the probe beam, proving that stimulated emission is the dominant process. Gain was also observed with a probe pulse length down to $10 \mathrm{ps}$ (data not shown).

Further tests were performed which are consistent with the STED mechanism: the temporal evolution of fluorescence quenching and signal gain were identical and matched the fluorescence decay of the dye. Secondly, the anisotropy of both the fluorescence emission and STED gain were found to coincide with the rotational correlation time, thus proving that the signal depends on the orientation of the dipole moment of the molecules to the polarization axis of the light. We checked also that both signals depend linearly on the laser power of the pump and the probe beam which is expected when measuring far away from saturation.

It would be preferable to analyze the two signals in absolute terms, i.e. to compare the number of photons lost in fluorescence and gained in the STED beam. This is difficult to achieve because it must be assured that the detection volume for both measurements is identical. This is not 
easily realizable experimentally and is subject to high uncertainties. Furthermore, the detection efficiencies are not exactly known and even vary for different measurements. Therefore both signals were measured relative to their unperturbed values (which is the signal from the total probe beam for the photon gain measurement and the full fluorescence signal in case for the quenching measurement). The detector efficiencies, especially the wavelength dependence of the two detectors, the PMT and the balanced detector, cancel out.

These relative measurements were performed for various wavelengths of the probe beam. In this way the experimental conditions remain constant, while the signal strength varies with the wavelength-dependent cross-section. The only correction needed is to account for the different focal spot size of each wavelength of the probe beam. For this correction, the beam waists were measured with the knife-edge method.

If stimulated emission were the quenching process, the spectral dependence of the fluorescence quenching would follow that of the cross section of stimulated emission $\sigma_{\mathrm{em}}$.

Two effects contribute to the signal change of the probe beam: stimulated emission $\sigma_{\mathrm{em}}$ and excited state absorption $\sigma^{*}$. Thus the gain signal is proportional to the difference of the two cross sections:

$P_{\text {probe }} \propto \sigma_{\mathrm{em}}-\sigma^{*}$.

Only in the case of a vanishing amount of excited state absorption does the gain signal follow the spectral dependence of $\sigma_{\mathrm{em}}$, given by [5]

$\sigma_{\mathrm{em}}(\lambda)=\frac{\lambda^{4} E(\lambda)}{8 \pi c n \tau}$,

with $c$ the speed of light in vacuum, $n$ the refractive index and $\tau$ the lifetime of the excited state $S_{n}$. The line shape function was determined from the measured fluorescence emission spectrum for the different dye solutions ( $Q=\int E(\lambda) \mathrm{d} \lambda$, where $Q$ is the quantum efficiency). The wavelength dependence of the employed spectrometer (Varian, Cary Eclipse, Palo Alto, USA) was taken into account. The result for every dye is depicted in Fig. 3 (thin line).

The results from the pump-probe measurements are depicted in the same graph. To associate a wavelength position for each data point the weighted mean of the transmission of each filter was determined. The results show that the fluorescence quenching $(\boldsymbol{\Delta})$ and the STED gain $(\boldsymbol{\square})$ have the same wavelength dependence for each dye within the accuracy of the measurement. Moreover, both follow the curve for the stimulated emission cross-section. A strong correlation between the STED gain, the fluorescence quenching and the stimulated emission cross-section was found. The three pairwise correlations were determined resulting in Pearson correlation coefficients between 0.973 and 0.9995 with a median of 0.997 .

These results are in very good agreement with the assumption that stimulated emission is responsible for the fluorescence quenching and that there is no substantial portion of excited state absorption. If there is excited state absorption, the wavelength dependence must follow that for stimulated emission, which would be extremely coincidental, or the cross-section for the $\mathrm{S}_{1}-\mathrm{S}_{\mathrm{n}}$ transition must be very small to be consistent within the accuracy of the measurement. A small probability of excited state absorption is essential for a good STED dye to minimize bleaching via higher excited states.

From the fact that we always observe a positive signal change in the probe beam and from Eq. (1) the conclusion can be drawn that $\sigma_{\mathrm{em}}>\sigma^{*}$. Because the up-conversion process is a cyclic-multiphotonic absorption process, many absorbed probe photons are needed to bring one excited molecule back to $\mathrm{S}_{0}$. Using an upper limit, one can assume that $10 \%$ of the molecules undergoing excited state absorption return radiationlessly to the ground state [11]. For the limiting case that both cross-sections for stimulated emission and excited state absorption are equal, which would mean that $50 \%$ of the molecules which are effected by the probe beam undergo stimulated emission and the other half excited state absorption, maximal $5 \%$ of these effected molecules would be quenched by an up-conversion process which would be $9 \%$ of all quenched molecules. That means, even for this limiting case, stimulated emission is the dominant process. Considering the good agreement of the measured data with the values for the stimulated emission cross-section, a more realistic ratio for the cross-sections would be $\sigma^{*} / \sigma_{\mathrm{em}}<10 \%$. From these numbers it follows that more than $99 \%$ of the quenched molecules are quenched by stimulated emission.

\section{Conclusion}

Pump-probe experiments were performed choosing probe wavelengths over a wide range of the emission spectra including those relevant for STED microscopy. We showed that photons are always gained in the probe beam after excitation to the $S_{1}$ state, while simultaneous quenching of fluorescence is observed for all fluorophores studied. Moreover, both signals follow the wavelength dependence of the stimulated emission cross-section. For these reasons, the absolutely dominant process for fluorescence quenching is stimulated emission. Up-conversion processes, for which the molecules have to be pumped multiple times to higher excited states thus reducing the number of photons in the probe beam, cannot be responsible for the strong fluorescence quenching responsible for breaking the diffraction barrier in fluorescence (STED) microscopy. This knowledge must be used to optimize the super-resolving microscope with respect to the depletion process.

\section{Acknowledgement}

We thank Christian Eggeling, Mariano Bossi and Lars Kastrup for helpful discussions and Andre Stiel for preparing the protein samples. 


\section{References}

[1] V. Westphal, S.W. Hell, Phys. Rev. Lett. 94 (14) (2005) 143903.

[2] G. Donnert et al., Proc. Natl. Acad. Sci. USA 103 (31) (2006) 11440.

[3] J.R. Lakowicz, I. Gryczynski, J. Kusba, V. Bogdanov, Photochem. Photobiol. 60 (6) (1994) 546.

[4] I. Gryczynski, J. Kusba, Z. Gryczynski, H. Malak, J.R. Lakowicz, J. Phys. Chem. 100 (24) (1996) 10135.

[5] O.G. Peterson, J.P. Webb, W.C. Mccolgin, J.H. Eberly, J. Appl. Phys. 42 (5) (1971) 1917.

[6] P. Didier, L. Guidoni, G. Schwalbach, M. Bourotte, A. FolleniusWund, C. Pigault, J.Y. Bigot, Chem. Phys. Lett. 364 (5-6) (2002) 503.

[7] K. Winkler, J.R. Lindner, V. Subramaniam, T.M. Jovin, P. Vohringer, Phys. Chem. Chem. Phys. 4 (6) (2002) 1072.

[8] Y. Iketaki et al., Chem. Phys. Lett. 372 (5-6) (2003) 773.
[9] Y. Iketaki, T. Watanabe, M. Sakai, S. Ishiuchi, M. Fujii, T. Watanabe, Opt. Eng. 44 (3) (2005) 033602.

[10] Y. Iketaki, T. Watanabe, N. Bokor, M. Fujii, Rev. Sci. Instrum. 77 (6) (2006) 063112.

[11] T. Watanabe et al., Chem. Phys. Lett. 420 (4-6) (2006) 410.

[12] J.C. Gumy, E. Vauthey, J. Phys. Chem. 100 (21) (1996) 8628.

[13] V.A. Alekseev, L.K. Denisov, V.I. Kozintsev, N.A. Kozlov, A.I. Sopin, J. Appl. Spectr. 31 (1) (1979) 51.

[14] I. Wieder, Appl. Phys. Lett. 21 (7) (1972) 318.

[15] B.G. Huth, G.I. Farmer, M.R. Kagan, J. Appl. Phys. 40 (13) (1969) 5145.

[16] K.I. Willig, S.O. Rizzoli, V. Westphal, R. Jahn, S.W. Hell, Nature 440 (7086) (2006) 935.

[17] K.I. Willig, R.R. Kellner, R. Medda, B. Hein, S. Jakobs, S.W. Hell, Nat. Method 3 (9) (2006) 721. 